\title{
ALTERNATIVAS PARA ATENUAR A DIFERENÇA DE ESTANDE NOS EXPERIMENTOS DE AVALIAÇÃO DE CLONES DE Eucalyptus urophylla ${ }^{1}$
}

Hélder Bolognani Andrade², Magno Antônio Patto Ramalho ${ }^{3}$, Júlio Sílvio de Souza Bueno Filho ${ }^{4}$, Marcos Deon Vilela de Resende ${ }^{5}$, Aloísio Xavier ${ }^{6}$ e José Roberto Soares Scolforo ${ }^{7}$

\begin{abstract}
RESUMO - Com o objetivo de estudar se as plantas adjacentes às falhas são capazes de compensar em parte ou totalmente as ausentes e se essa compensação varia com o clone e as condições edafoclimáticas, foi conduzido o presente trabalho, em dois municípios da região noroeste do Estado de Minas Gerais, sendo os experimentos implantados em dezembro de 1998. O delineamento adotado foi o de blocos as acaso, no esquema de parcelas subdivididas, com três repetições. A parcela foi constituída por sete diferentes clones e a subparcela, pelo número variável de plantas no estande, em função do porcentual de falha. A subparcela que não apresentou falha recebeu 10 plantas em linha. Para a constituição das demais subparcelas, foram adotados cinco diferentes níveis de falha $(10,20,30,40$ e $50 \%)$. Aos 37 meses de idade, foi avaliado o volume de madeira ( $\mathrm{m}^{3}$ sólido/ ha). Realizaram-se análises de variância por local e, posteriormente, análises conjuntas de locais, em ambos os casos envolvendo os dados no nível de média de planta e total de parcela. Nas análises de variância, as fontes de variação que envolveram a variável estande foram decompostas em efeitos lineares, quadráticos e desvios da regressão. Os valores de b foram estimados para estande e para cada clone, em cada local, independentemente do clone. A partir dos resultados obtidos, pôde-se concluir que: a maioria dos clones avaliados não compensa, em parte, a ausência das plantas vizinhas; a capacidade de compensação difere entre os clones e as condições edafoclimáticas; independentemente do clone e local de avaliação, a correção do estande por regressão e, principalmente, por co-variância foi eficiente para efetuar a seleção dos melhores clones, sendo mais expressiva com os dados de total de parcela.
\end{abstract}

Palavras-chave: Eucalyptus urophylla, clone, estande e falha.

\section{ALTERNATIVE TO ATTENUATE PLANT STAND DIFFERENCE IN EXPERIMENTS OF EUcalyptus urophylla CLONE ASSESSMENT}

\begin{abstract}
A study to investigate whether plants adjacent to failed plants are able to partially or completely compensate the absent plants, and whether this compensation depends on clone and edaphic-climatic conditions was carried out in two northwestern Minas Gerais counties. The experiments were set up in December 1998. A randomized complete block design with split plot treatments and three replications was used. The plots consisted of seven different clones and the split plot was the variable number of plants in the stand, as a function of the failure percentage. The split plot that did not present any failure had 10 plants in a row. To constitute the other plots, five different failure levels were adopted (10, 20, 30, 40 and 50\%). The assessed trait was wood volume (solid $\mathrm{m}^{3} / \mathrm{ha}$ ) at 37 months of age. Individual analyses of variance were performed
\end{abstract}

\footnotetext{
${ }^{1}$ Recebido em 09.01.2003 e aceito para publicação em 10.11.2005.

${ }^{2}$ V\&M Florestal, Praça Voluntários da Pátria, 81 - Centro - 35790-000 Curvelo-MG. E-mail: <helderb@vmtubes.com.br>.

${ }^{2}$ Departamento de Biologia, Universidade Federal de Lavras -UFLA, Cx.P. 37. 37200-000 Lavras-MG.

${ }^{3}$ Departamento de Ciências Exatas, UFLA, Cx.P. 37. 37200-000 Lavras, MG.

${ }^{4}$ EMBRAPA-CNPF, Cx.P. 23.34444-444 Colombo-PR.

${ }^{5}$ Departamento de Engenharia Florestal, Universidade Federal de Viçosa. 36570-000 Viçosa-MG.

${ }^{6}$ Departamento de Ciências Florestais, UFLA, Cx.P. 37. 37200-000 Lavras-MG.
} 
for each location. Joint analyses of location were also performed. Both types of analyses involved data at the plant mean and plot total levels. Sources of variation that involved the variable stand were partitioned into linear and quadratic effects and deviation from the regression. The regression $b$ values were estimated for stand, individual clone and location regardless of the clone. The following conclusions were obtained from the results: a) most of the assessed clones did not totally compensate the absence of the neighboring plants; b) the compensation capacity varies with clones and edaphic-climatic conditions; c) regardless of the clone or location, stand correction by regression, and especially by covariance, improved the clone selection efficiency, in particular with the total plot data.

Keywords: Eucalyptus urophylla, clone, stand and failure.

\section{INTRODUÇÃO}

Em qualquer programa de melhoramento, a etapa de avaliação é a mais cara e demorada. Os experimentos devem ser conduzidos com o maior rigor possível para que tenham boa precisão e, assim, as diferenças fenotípicas representem as genotípicas. Há vários fatores que afetam a precisão experimental, como a heterogeneidade do solo, o tamanho da parcela, o delineamento adotado, o número de repetições e a condução dos experimentos (MARQUES JÚNIOR, 1997; STEEL et al., 1997; RAMALHO et al., 2000).

Outro fator que afeta o desempenho dos experimentos e, muitas vezes, está fora do controle do pesquisador é a perda de plantas por ataque de insetos, pragas e danos mecânicos. Essa perda de plantas é normalmente aleatória e acarreta diferença no estande, o que evidentemente contribui para a redução da precisão experimental (VERONESI et al., 1995). No caso das espécies anuais cultivadas e de importância econômica, há relatos do efeito do estande e, sobretudo, do modo de atenuá-lo (FERNANDES et al., 1989; SCHMILDT, 2000). Contudo, em se tratando de plantas perenes, em que normalmente as parcelas experimentais envolvem menor número de indivíduos e as perdas de algumas plantas podem ter o efeito mais expressivo, as informações são escassas.

Na avaliação de clones de eucalipto, a ocorrência de estandes heterogêneos é comum, sobretudo devido a diferenças no vigor das mudas, além daquelas já mencionadas anteriormente. Nessa condição, é questionável se plantas adjacentes às falhas são capazes de compensar, em parte ou totalmente, as ausentes e, mais ainda, se essa compensação varia com o clone, a idade de avaliação e as condições edafoclimáticas.

Para responder a esses questionamentos e propor alternativas para atenuar as diferenças de estande na avaliação de clones, foi realizado o presente trabalho.

\section{MATERIAL E MÉTODOS}

\subsection{Locais}

Os testes foram implantados nos Municípios de Paraopeba e Bocaiúva, no Estado de Minas Gerais, os quais apresentam características edafoclimáticas contrastantes (Quadro 1). Conforme o zoneamento ecológico realizado por Golfari (1975), são pertencentes a região bioclimática 7 .

\subsection{Material Experimental}

Para a condução deste teste foram selecionados sete clones, originados de híbridos naturais de Eucalyptus urophylla, que foram ou são utilizados para plantio na região noroeste do Estado de Minas Gerais.

Quadro 1 - Características edafoclimáticas e localização geográfica dos locais de instalação dos testes

Table 1 - Edaphic-climatic characteristics and geographic localization of the test installation sites

\begin{tabular}{lccc}
\hline Características & \multicolumn{2}{c}{ Locais } \\
& & Paraopeba & Bocaiúva \\
\hline Latitude (S) & $17^{\circ} 20^{\prime}$ & $19^{\circ} 17^{\prime}$ \\
Longitude (W) & $43^{\circ} 20^{\prime}$ & $44^{\circ} 29^{\prime}$ \\
Altitude (m) & 700 & 850 \\
Precipitação (mm) & 1350 & 850 \\
Déficit hídrico (mm) & $40-100$ & $90-210$ \\
Solo & Areia & 4,7 & 14,3 \\
Física (\%) & Silte & 19,2 & 17,6 \\
& Argila $^{2}$ & 76,1 & 68,1 \\
Química & $\mathrm{Ca}^{+2}\left(\mathrm{cmol} / \mathrm{dm}^{3}\right)$ & 0,32 & 0,52 \\
& $\mathrm{Mg}^{+2}\left(\mathrm{cmol} / \mathrm{dm}^{3}\right)$ & 0,05 & 0,09 \\
& $\mathrm{~K}^{+}\left(\mathrm{mg} / \mathrm{dm}^{3}\right)$ & 45,21 & 24,70 \\
\hline
\end{tabular}




\subsection{Instalação e Condução dos Ensaios}

Os experimentos foram implantados nos dois locais, em dezembro de 1998. O delineamento adotado foi o de blocos ao acaso, no esquema de parcelas subdivididas, com três repetições. A parcela foi constituída pelos clones e a subparcela, pelo número variável de plantas no estande, em função do porcentual de falha; a subparcela que não apresentou falha recebeu 10 plantas em linha. Para a constituição das demais subparcelas, foram adotados cinco diferentes níveis de falha (10, 20, 30, 40 e 50\%). Para a simulação das falhas, foi realizado um sorteio prévio das plantas a serem eliminadas em cada situação. As eliminações das plantas nas subparcelas que apresentavam falhas foram realizadas três meses após o plantio. Para evitar o efeito da competição lateral nas subparcelas, foram mantidas subparcelas sem falha, alternadas por subparcelas que receberam os tratamentos propostos. Isso possibilitou a formação de uma bordadura sem falha, em torno das subparcelas que receberam os tratamentos descritos anteriormente.

As mudas foram produzidas em tubetes, as quais apresentavam idade entre 90 e 110 dias. O preparo do solo foi realizado com grade "bedding” em nível. O espaçamento adotado foi de 3,0 m entre linhas x 3,0 $\mathrm{m}$ entre plantas. A adubação de plantio foi realizada com $200 \mathrm{~kg} /$ ha de super simples mais 1\% de boro, aplicado em sulco na linha de plantio. Aos 10 meses de idade foram aplicados, a lanço, calcário e cloreto de potássio. As doses dos fertilizantes aplicados em cada local foram realizadas com base nos resultados das análises de solo.

\subsection{Caracteres Avaliados}

Nos dois experimentos foram coletados dados em nível de planta por parcela, para as características altura total (m) e circunferência à altura do peito - CAP $(\mathrm{cm})$, aos 37 meses de idade. Para o cálculo do volume de madeira ( $\mathrm{m}^{3}$ sólido/ha), foram cubadas, de metro em metro, três árvores por repetição, para cada um dos clones. Com os resultados dessa cubagem, foram estimadas equações de regressão para volume sólido em função do CAP e da altura total. Com essas equações foram estimados os volumes de madeira de cada parcela.

\subsection{Análises Estatísticas}

Foram realizadas análises de variância da característica volume de madeira no nível de média de planta e total de parcela. Primeiramente, realizou-se uma análise em nível de local. Todos os efeitos do modelo foram considerados fixos, exceto blocos e o erro, conforme Stell et al. (1997).

$$
y_{i j s}=m+t_{i}+b_{j}+(t b)_{i j}+s_{s}+(t s)_{i s}+\bar{e}_{i j s}
$$

em que:

$\mathrm{y}_{\mathrm{ijs}}=$ valor observado no bloco j do clone i no número s de falhas;

$\mathrm{m}=$ média geral;

$\mathrm{t}_{\mathrm{i}}=$ efeito fixo do i-ésimo clone; $\mathrm{i}=1,2, \ldots, \mathrm{n}$;

$\mathrm{b}_{\mathrm{j}}=$ efeito aleatório do j-ésimo bloco; $\mathrm{j}=1,2, \ldots, \mathrm{r}$;

$(\mathrm{tb})_{\mathrm{ij}}=$ efeito da interação entre os clones i com o bloco j;

$\mathrm{s}_{\mathrm{s}}=$ efeito do número $\mathrm{s}$ de falhas; $\mathrm{s}=1,2, \ldots, \mathrm{k}$;

$(t s)_{\text {is }}=$ efeito da interação entre os clones i com o número s de falhas; e

$\mathrm{e}_{\mathrm{ijs}}=$ erro experimental, sendo eijs $\cap \mathrm{N}(0, \sigma 2)$.

Posteriormente, foram realizadas as análises conjuntas de locais envolvendo novamente os dados no nível de média de planta e total de parcela, adotandose o seguinte modelo estatístico e considerando todos os efeitos fixos, exceto blocos e o erro:

$$
\begin{aligned}
y_{i j q s}= & m+t_{i}+r_{j(q)}+a_{q}+(t a)_{i q}+(t r)_{i j(q)}+ \\
& +s_{s}+(t s)_{i s}+(a s)_{q s}+(t a s)_{i q s}+\bar{e}_{i j q s}
\end{aligned}
$$

em que:

$\mathrm{y}_{\mathrm{ijqs}}=$ valor observado do bloco j dentro do local q, do clone i, do número de falhas s;

$\mathrm{m}=$ média geral;

$\mathrm{t}_{\mathrm{i}}=$ efeito do i-ésimo clone; $\mathrm{i}=1,2, \ldots, \mathrm{n}$;

$\mathrm{rj}_{\mathrm{(q)}}=$ efeito do bloco $\mathrm{j}$ dentro do local $\mathrm{q} ; \mathrm{j}=1,2, \ldots, \mathrm{r}$;

$\mathrm{a}_{\mathrm{q}}=$ efeito do local q; $\mathrm{q}=1,2$;

$(\mathrm{ta})_{\mathrm{iq}}=$ efeito da interação entre os clones i e os locais q;

$(\mathrm{tr})_{\mathrm{ij}(\mathrm{q})}=$ efeito da interação entre os clones i com o bloco j, dentro dos locais q;

$\mathrm{s}_{\mathrm{s}}=$ efeito do número $\mathrm{s}$ de falhas; $\mathrm{s}=1,2, \ldots, \mathrm{k}$;

$(\text { ts })_{\text {is }}=$ efeito da interação entre os clones i com o número s de falhas;

(as) $)_{\mathrm{qs}}=$ efeito da interação entre os locais q com o número s de falhas;

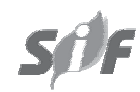

R. Árvore, Viçosa-MG, v.30, n.1, p.11-18, 2006 
$(\text { tas })_{\text {iq̣s }}=$ efeito da interação entre os clones i com os locais q e com o número s de falhas; e

$\mathrm{e}_{\mathrm{ijqs}}=$ erro experimental, sendo eijqs $\cap \mathrm{N}(0, \sigma 2)$.

Na análise de variância, as fontes de variação que envolveram a variável estande foram decompostas em efeitos lineares, quadráticos e desvios da regressão. Os valores de b foram estimados, para cada clone ou cada local, independentemente do clone. A partir dessas estimativas, foram obtidos os volumes médio e total de madeira corrigidos (yc) pela expressão:

$\mathrm{Yc}=\mathrm{y}-\mathrm{b}(\mathrm{x}-10)$, em que y é o valor referente ao dado da parcela, b é o coeficiente de regressão linear para cada clone ou local e x é o número de plantas na parcela. O modelo de análise de variância corrigido pelas estimativas dos bs foi o mesmo comentado anteriormente. Nesse caso, também foi realizada a análise com os dados sem correção, para verificar a eficiência da regressão como fator de correção do estande.

\section{RESULTADOS E DISCUSSÃO}

Na implantação e condução dos experimentos de campo são muitos os fatores que o pesquisador deve procurar controlar. Dentre eles estão as falhas que levam à desuniformidade no estande, causando dificuldades na condução e avaliação dos experimentos, sendo um dos problemas básicos na análise e interpretação dos resultados experimentais. Esse fator émais agravante em culturas perenes, visto que os erros podem ser cumulativos e perpetuarem durante toda a condução do ensaio.
Os resumos das análises de variância por local de avaliação, considerando-se dados médios por planta ou total por parcela, estão apresentados no Quadro 2. Verificou-se que ocorreu diferença significativa entre clones $(P \leq 0,01)$ em todas as situações, evidenciando, como esperado, que os clones eram diferentes, condição essa fundamental para a realização de trabalho desta natureza. Como a perda de plantas por parcela foi induzida, seria esperada uma diferença significativa entre os estandes; em alguns casos, isso veio a ocorrer, por exemplo, na avaliação do volume médio de madeira por planta.

No caso da interação, esta foi significativa ( $\mathrm{P} \leq$ $0,01)$ somente nos dados de volume total, indicando que, nessa situação, o efeito do estande não foi coincidente com o clone avaliado. Esses resultados, em princípio, já evidenciam a dificuldade de atenuar os efeitos da diferença do número de plantas por parcela, pois o seu efeito varia com o clone, com o local e o tipo de unidade utilizada nas análises.

No Quadro 3 é apresentado o resumo da análise de variância conjunta de locais, considerando-se como unidade o volume por planta ou total da parcela. Novamente, o efeito de clones foi significativo $(\mathrm{P} \leq$ $0,01)$ em todos os casos, o mesmo ocorrendo com locais. Contudo, a interação clones $x$ locais foi significativa $(\mathrm{P} \leq 0,01)$ em todos os casos, evidenciando-se que o comportamento dos clones não foi coincidente nos dois locais de avaliação.

Quadro 2 - Resumo da análise de variância do volume de madeira ( $\mathrm{m}^{3}$ sólido/ha), utilizando-se dados médios por planta ou total de parcela, obtido em experimentos de avaliação de clones de eucalipto em diferentes estandes, avaliado aos 37 meses em Paraopeba e Bocaiúva, MG

Table 2 - Summary of variance analysis of wood volume $\left(\mathrm{m}^{3}\right.$ solid/ha), using data at the plant mean and plot total levels, obtained from experiments for evaluation of eucalyptus clones in different stands, at 37 months old in Paraopeba and Bocaiúva, $M G$

\begin{tabular}{|c|c|c|c|c|c|}
\hline \multirow[t]{3}{*}{$\mathrm{FV}$} & \multirow{3}{*}{ GL } & \multicolumn{4}{|c|}{ QM } \\
\hline & & \multicolumn{2}{|c|}{ Média } & \multicolumn{2}{|c|}{ Total } \\
\hline & & Paraopeba & Bocaiúva & Paraopeba & Bocaiúva \\
\hline Repetição & 2 & 2014,63 & 131,12 & 80458,92 & 4129,22 \\
\hline Clone (C) & 6 & $24064,76 * *$ & $5684,55 * *$ & $1267367,37 * *$ & $316563,17 * *$ \\
\hline Erro 1 & 12 & 614,12 & 82,11 & 17677,61 & 1999,81 \\
\hline Estande (E) & 5 & $922,95 * *$ & $93,30 *$ & 9096,50 n.s. & 733,24 n.s. \\
\hline$C \times E$ & 30 & 208,02 n.s. & 45,77 n.s. & $21206,11 * *$ & $5960,09 * *$ \\
\hline Erro 2 & 70 & 133,82 & 31,81 & 6099,55 & 1368,57 \\
\hline Média ( $\mathrm{m}^{3}$ sol./ha) & & 88,72 & 44,03 & 833,27 & 421,67 \\
\hline CV $1(\%)$ & & 27,93 & 20,58 & 15,96 & 10,61 \\
\hline CV 2 (\%) & & 13,04 & 12,81 & 9,37 & 8,77 \\
\hline
\end{tabular}

* e ** significativos pelo teste $\mathrm{F}$ a 5 e $1 \%$ de probabilidade, respectivamente; e n.s.: não-significativo. 
Quadro 3-Resumo da análise de variância conjunta do volume de madeira ( $\left.\mathrm{m}^{3} \mathrm{sol} . / \mathrm{ha}\right)$, utilizando-se os dados médios por planta e total de parcela, obtidos em experimentos de avaliação de clones de eucalipto em diferentes estandes e locais, avaliado aos 37 meses de idade

Table 3 - Summary of joint variance analysis of wood volume $\left(\mathrm{m}^{3} \mathrm{solid} / \mathrm{ha}\right)$, using data at the plant mean and plot total levels, obtained from experiments for evaluation of eucalyptus clones in different stands and sites, at 37 months old

\begin{tabular}{|c|c|c|c|}
\hline \multirow[t]{2}{*}{ FV } & \multirow[t]{2}{*}{ GL } & \multicolumn{2}{|c|}{ QM } \\
\hline & & Média & Total \\
\hline Repetição/local & 4 & $686,80 *$ & $42275,57 * *$ \\
\hline Clone (C) & 6 & $24236,75 * *$ & $1344875,55 * *$ \\
\hline Local (L) & 1 & $133229,81 * *$ & $7160846,85 * *$ \\
\hline C x L & 6 & $4302,55 * *$ & $239064,49 * *$ \\
\hline Erro 1 & 24 & 175,76 & 9837,49 \\
\hline Estande (E) & 5 & $716,80 * *$ & $389041,79 * *$ \\
\hline Linear & 1 & $3336,75 * *$ & $1925366,01 * *$ \\
\hline Quadrática & 1 & 3,33 n.s. & 3156,15 n.s. \\
\hline Desvio & 3 & 81,31 n.s. & 5562,27 n.s. \\
\hline $\mathrm{E} \times \mathrm{C}$ & 30 & $132,00 * *$ & $18876,40 * *$ \\
\hline E/Clone 1 & 5 & $403,67 *$ & $158216,02 * *$ \\
\hline Linear & 1 & $820,40 *$ & $720616,87 * *$ \\
\hline Quadrática & 1 & 336,96 n.s. & 17923,87 n.s. \\
\hline Desvio & 3 & 286,99 n.s. & 17513,12 n.s. \\
\hline E/Clone 2 & 5 & 41,88 n.s. & $23269,21 *$ \\
\hline Linear & 1 & 27,57 n.s. & $103368,85 * *$ \\
\hline Quadrática & 1 & 12,01 n.s. & 1100,64 n.s. \\
\hline Desvio & 3 & 56,61 n.s. & 3958,85 n.s. \\
\hline E/Clone 3 & 5 & $463,49 * *$ & $39732,47 * *$ \\
\hline Linear & 1 & $1759,31 * *$ & $151149,16 * *$ \\
\hline Quadrática & 1 & 7,75 n.s. & 6510,25 n.s. \\
\hline Desvio & 3 & 183,45 n.s. & 13667,64 n.s. \\
\hline E/Clone 4 & 5 & 203,92 n.s. & $32137,92 * *$ \\
\hline Linear & 1 & 278,16 n.s. & $110403,07 * *$ \\
\hline Quadrática & 1 & 401,25 n.s. & $30627,49 *$ \\
\hline Desvio & 3 & 113,40 n.s. & 6553,01 n.s. \\
\hline E/Clone 5 & 5 & 221,76 n.s. & $133907,99 * *$ \\
\hline Linear & 1 & $737,36 *$ & $646314,25 * *$ \\
\hline Quadrática & 1 & 9,26 n.s. & 1634,40 n.s. \\
\hline Des & 3 & 120,73 n.s. & 7197,11 n.s. \\
\hline E/Clone 6 & 5 & 32,79 n.s. & $97327,65 * *$ \\
\hline Linear & 1 & 90,54 n.s. & $481326,69 * *$ \\
\hline Quadrática & 1 & 10,63 n.s. & 389,73 n.s. \\
\hline Desvio & 3 & 20,94 n.s. & 1640,61 n.s. \\
\hline E/Clone 7 & 5 & 141,34 n.s. & $17708,90 *$ \\
\hline Linear & 1 & $559,14 *$ & $79532,80 * *$ \\
\hline Quadrática & 1 & 41,60 n.s. & 731,04 n.s. \\
\hline Desvio & 3 & 35,32 n.s. & 2760,23 n.s. \\
\hline$E \times L$ & 10 & $204,21 * *$ & $42533,77 * *$ \\
\hline E/Local1 & 5 & $805,70 * *$ & $338999,81 * *$ \\
\hline Linear & 1 & $3367,77 * *$ & $1650216,73 * *$ \\
\hline Quadrática & 1 & 20,70 n.s. & 732,73 n.s. \\
\hline Desvio & 3 & 213,34 n.s. & 14683,20 n.s. \\
\hline E/Local 2 & 5 & 115,32 n.s. & $92575,75 * *$ \\
\hline Linear & 1 & $559,75 *$ & $459305,20 * *$ \\
\hline Quadrática & 1 & 3,88 n.s. & 2743,76 n.s. \\
\hline Desvio & 3 & 4,32 n.s. & 276,59 n.s. \\
\hline$E \times C \times L$ & 30 & $111,17 *$ & $8292,55 * *$ \\
\hline Erro 2 & 140 & 64,12 & 3734,00 \\
\hline Média & & 67,74 & 500,59 \\
\hline CV $1(\%)$ & & & \\
\hline CV 2 (\%) & & 11,82 & 12,21 \\
\hline
\end{tabular}

* $\mathrm{e} * *$ significativos pelo teste $\mathrm{F}$ a 5 e $1 \%$ de probabilidade, respectivamente; e n.s.: não-significativo.
O objetivo principal deste trabalho foi verificar o efeito do estande. Como era esperado, pelas razões já apontadas, detectou-se diferença significativa $(\mathrm{P} \leq 0,01)$ nessa fonte de variação, considerando as análises tanto com média quanto com totais (Quadro 3). Como interessava verificar se o efeito do número de falha era linearmente relacionado ao desempenho do clone, o efeito de estande foi decomposto em respostas linear e quadrática. Observou-se que em todos os casos o desvio da regressão foi não-significativo, indicando que, com uma equação linear ou quadrática, é possível ter um bom ajuste da resposta do volume de madeira em função da diferença do número de plantas por parcela. Na maioria dos casos, independentemente do clone e do local, a resposta foi linear. Em princípio, essa é uma situação favorável para se atenuar o efeito da diferença de estande.

A ocorrência da interação clones x locais é evidenciada pelos resultados médios apresentados no Quadro 4. A classificação dos clones variou entre os locais. Chamaram atenção os clones 1 e 5 , que foram os de melhor desempenho em todas as situações; e os clones 2 e 4, que foram os de pior desempenho. Interações envolvendo esses ambientes e materiais genéticos também já foram detectadas por Andrade et al. (1994).

Como a interação clones $\mathrm{x}$ estandes foi significativa ( $\mathrm{P} \leq 0,01)$, decompôs-se o efeito de estande dentro de cada clone (Quadro 3). Quando se considerou como unidade o volume médio por planta, não houve efeito de estande, exceto nos clones 1 e 3 . Porém, quando a análise foi efetuada com totais, todos os clones foram afetados pelo estande e responderam linearmente ao número de plantas perdidas na parcela.

Quadro 4 - Produção de madeira no nível de média de planta e total de parcela $\left(\mathrm{m}^{3}\right.$ solido/ha) dos diferentes clones aos 37 meses, em Paraopeba e Bocaiúva

Table 4 - Wood production at the plant mean and plot total levels ( $\mathrm{m}^{3}$ solid/ha), for the different clones at 37 months old, in Paraopeba and Bocaiúva

\begin{tabular}{crrrrr}
\hline Clones & \multicolumn{2}{c}{ Média } & & \multicolumn{2}{c}{ Total } \\
\cline { 2 - 3 } \cline { 6 - 6 } \cline { 5 - 6 } & Paraopeba & Bocaiúva & & Paraopeba & Bocaiúva \\
\hline 1 & $137,77 \mathrm{a}$ & $67,81 \mathrm{a}$ & & $1010,41 \mathrm{a}$ & $509,03 \mathrm{a}$ \\
2 & $47,42 \mathrm{c}$ & $25,83 \mathrm{~d}$ & & $347,36 \mathrm{c}$ & $194,14 \mathrm{~d}$ \\
3 & $102,70 \mathrm{~b}$ & $35,58 \mathrm{c}$ & & $751,92 \mathrm{~b}$ & $256,81 \mathrm{c}$ \\
4 & $62,17 \mathrm{c}$ & $27,97 \mathrm{~d}$ & & $461,09 \mathrm{c}$ & $207,71 \mathrm{~d}$ \\
5 & $122,97 \mathrm{a}$ & $68,98 \mathrm{a}$ & & $906,68 \mathrm{a}$ & $512,64 \mathrm{a}$ \\
6 & $100,51 \mathrm{~b}$ & $44,75 \mathrm{~b}$ & & $753,91 \mathrm{~b}$ & $332,86 \mathrm{~b}$ \\
7 & $47,52 \mathrm{c}$ & $37,28 \mathrm{c}$ & & $341,83 \mathrm{c}$ & $277,08 \mathrm{c}$ \\
\hline
\end{tabular}

Médias seguidas da mesma letra na coluna não diferem entre si, pelo Teste de Scott-Knott ( $\mathrm{P} \leq 0,05)$. 
Estimou-se o coeficiente de regressão linear (b), que mede a resposta no volume de madeira em função da alteração no número de plantas por parcela (Quadro 5). Corroborando o resultado da análise de variância quando se considera o volume médio por planta, a estimativa de b só foi expressiva no clone 3, o único em que ocorreu efeito significativo de estande. A estimativa de b corresponde a 5,92\% da média, ou seja, para esse clone, cada planta em falta na parcela contribuiu para o incremento médio do volume por planta de 5,92\% do seu desempenho médio.

Quando se considera total de parcela (Quadro 5), há aumento no valor do coeficiente de determinação $\left(\mathrm{R}^{2}\right)$, bem como de b, em comparação com os valores obtidos com média. No caso do coeficiente de regressão, este foi negativo, como esperado, haja vista que a perda de plantas contribuiu evidentemente para a redução da produtividade total da parcela. É interessante salientar que a estimativa de $b$ do clone 3 foi de pequena magnitude, o que indica que o efeito da falha no volume total, nesse clone, é menos pronunciado. Isso é fácil de explicar porque, como visto anteriormente, esse clone tem a capacidade de compensar a falha, por meio de maior crescimento das plantas vizinhas. Essa foi a razão da interação clones x estandes, reforçando o que já foi comentado de que a atenuação do efeito de estande é dificultada pelo fato de não se poder ter um ajuste único em todos os clones.

A interação estandes $\mathrm{x}$ locais foi significativa ( $\mathrm{P}$ $\leq 0,01)$. Contudo, dentro de cada local, independentemente do clone, foi sempre linear. Observa-se, no Quadro 5 , que o coeficiente de regressão foi de magnitude diferente nos dois locais. Em Bocaiúva, onde as condições edafoclimáticas são menos favoráveis (Quadro 1), os valores de b são de menor magnitude, isto é, a compensação da falha é função da condição ambiental. Isso ocorreu tanto para volume por planta quanto para volume total.

Para comprovar que a estimativa de b pode ser utilizada como um fator de correção do estande, foram realizadas novas análises de variância, empregandose o ajuste do dado por planta e no total e considerando um coeficiente de regressão b médio, independentemente do clone e local. Posteriormente, foi realizada a mesma análise, utilizando-se como fator de correção o b estimado para cada clone e repetindo o mesmo procedimento em cada local. Os valores de b usados estão apresentados no Quadro 5. Verifica-se que, nesse caso, o ajuste foi bom, pois o efeito de estande foi não-significativo, tanto para volume por planta quanto para volume total (Quadros 6 e 7). Deve ser enfatizado, todavia, que as interações envolvendo estande continuaram sendo significativas, como era esperado.

Na literatura há inúmeros relatos de procedimentos que podem ser utilizados para a correção de estande com plantas anuais (CRUZ, 1971; VERONESI et al., 1995; SCHMILDT, 2000), entretanto não foi encontrado nenhum relato da correção de estande em plantas perenes. Nessa situação, como o número de plantas por parcela é normalmente menor, o efeito do estande deve ser mais acentuado.

Quadro 5 - Estimativas dos coeficientes de regressão (b), coeficientes de determinação (R2) e porcentual de crescimento volumétrico em relação à média (A), obtidos para estande, clones e locais, com os dados em nível de média de planta e total de parcela, avaliados na idade de 37 meses

Table 5 - Estimates of regression coefficient (b), determination coefficient ( $R 2)$ and percentage of volumetric growth in relation to the mean (A), obtained for stand, clones and sites, with data at the plant mean and plot total levels, at 37 months old

\begin{tabular}{|c|c|c|c|c|c|c|}
\hline \multirow[t]{2}{*}{ Parâmetro } & \multicolumn{3}{|c|}{ Média } & \multicolumn{3}{|c|}{ Total } \\
\hline & $\mathrm{R}^{2}(\%)$ & $\mathrm{b}$ & A (\%) & $\mathrm{R}^{2}(\%)$ & $\mathrm{b}$ & A (\%) \\
\hline Estande & 93,10 & 2,1307 & & 98,98 & $-51,1815$ & \\
\hline Clone 1 & 40,65 & 2,7952 & 2,72 & 91,09 & $-82,8433$ & 10,90 \\
\hline Clone 2 & 13,16 & 0,5124 & 1,40 & 88,85 & $-31,3762$ & 11,59 \\
\hline Clone 3 & 75,92 & 4,0933 & 5,92 & 76,08 & $-37,9410$ & 7,52 \\
\hline Clone 4 & 27,28 & 1,6276 & 3,61 & 68,71 & $-32,4262$ & 9,70 \\
\hline Clone 5 & 66,50 & 2,6500 & 2,76 & 96,53 & - 78,4562 & 11,06 \\
\hline Clone 6 & 55,21 & 0,9286 & 1,28 & 98,91 & $-67,7057$ & 12,46 \\
\hline Clone 7 & 79,12 & 2,3076 & 5,44 & 89,82 & - 27,5219 & 8,89 \\
\hline Paraopeba & 83,60 & 3,0272 & & 97,36 & - 67,0103 & \\
\hline Bocaiúva & 97,08 & 1,2342 & & 99,23 & $-35,3524$ & \\
\hline
\end{tabular}

R. Árvore, Viçosa-MG, v.30, n.1, p.11-18, 2006 
Quadro 6 - Resumo da análise de variância conjunta do volume de madeira ( $\mathrm{m}^{3}$ sólido/ha), utilizando-se dados médios por planta sem correção e com correção, para efeito de estande, efeito de estande dentro de clone e efeito de estande dentro de local, obtido em experimentos de avaliação de clones de eucalipto em diferentes estandes, avaliado aos 37 meses de idade

Table 6 - Summary of joint variance analysis of the wood volume ( $\mathrm{m}^{3}$ solid/ha), using mean data per plant with and without correction, for stand effect, stand effect inside clones and stand effect inside site, obtained from experiments for evaluation of eucalyptus clones in different stands, at 37 months old

\begin{tabular}{lccccc}
\hline \multicolumn{1}{c}{ FV } & GL & Sem correção & Cor/Estande & Cor/Clone & Cor/Local \\
\hline Repetição/Local & 4 & $686,81^{*}$ & $686,33 *$ & $686,33 *$ & $686,31 *$ \\
Clone (C) & 6 & $24236,75^{* *}$ & $24238,84^{* *}$ & $21933,44^{* *}$ & $24238,67 * *$ \\
Local (L) & 1 & $133229,81^{* *}$ & $133209,57 * *$ & $133204,52 * *$ & $108501,33 * *$ \\
C x L & 6 & $4302,55^{* *}$ & $4301,06 * *$ & $4300,86 * *$ & $4301,13 * *$ \\
Erro 1 & 24 & 175,76 & 175,94 & 175,96 & 175,94 \\
Estande (E) & 5 & $716,80^{* *}$ & 49,14 n.s. & 49,16 n.s. & 49,18 n.s. \\
E x C & 30 & $132,01 * *$ & $132,00 * *$ & $100,76 *$ & $132,00 * *$ \\
E x L & 10 & $204,21 * *$ & $204,31^{* *}$ & $204,26 * *$ & 85,96 n.s. \\
E x C x L & 30 & $111,17^{*}$ & $111,12 *$ & $111,11 *$ & $111,16 *$ \\
Erro 2 & 140 & 64,12 & 64,09 & 64,09 & 64,09 \\
\hline Média (m ${ }^{3}$ sólido/ha) & & 67,74 & 62,41 & 62,41 & 62,41 \\
CV 1 (\%) & & 19,57 & 21,25 & 21,25 & 21,25 \\
CV 2 (\%) & 11,82 & 12,83 & 12,83 & 12,83 \\
\hline
\end{tabular}

* e ** significativos pelo teste $\mathrm{F}$ a 5 e $1 \%$ de probabilidade, respectivamente; e n.s.: não-significativo.

Quadro 7 - Resumo da análise de variância conjunta do volume de madeira ( $\mathrm{m}^{3}$ sólido/ha), utilizando-se dados de total de parcela sem correção e com correção, para efeito de estande, efeito de estande dentro de clone e efeito de estande dentro de local, obtidos em experimentos de avaliação de clones de eucalipto em diferentes estandes, avaliado aos 37 meses de idade

Table 7 - Summary of joint variance analysis of the wood volume ( $m^{3}$ solid/ha), using data at the plot total level with and without correction, for stand effect, stand effect inside clones and stand effect inside site, obtained from experiments for evaluation of eucalyptus clones in different stands, at 37 months old

\begin{tabular}{lccccr}
\hline \multicolumn{1}{c}{ FV } & GL & Sem correção & Cor/Estande & Cor/Clone & Cor/Local \\
\hline Repetição/Local & 4 & $42275,57 * *$ & $42288,99 * *$ & $42289,30^{* *}$ & $42288,62 * *$ \\
Clone (C) & 6 & $1344875,55^{* *}$ & $1344892,41 * *$ & $2273501,67 * *$ & $1344891,08 * *$ \\
Local (L) & 1 & $7160846,85 * *$ & $7160870,45 * *$ & $7160914,28 * *$ & $10917550,77 * *$ \\
C x L & 12 & $239064,49^{* *}$ & $239045,09 * *$ & $239045,03 * *$ & $239046,08 * *$ \\
Erro 1 & 24 & 9837,49 & 9839,27 & 9839,04 & 9839,25 \\
Estande (E) & 5 & $389041,79 * *$ & 3969,77 n.s. & 3969,78 n.s. & 3969,41 n.s. \\
E x C & 30 & $18876,40 * *$ & $18875,39^{* *}$ & $6631,44^{*}$ & $18875,49 * *$ \\
E x L & 10 & $42533,77 * *$ & $42533,70 * *$ & $42533,12 * *$ & 5701,81 n.s. \\
E x C x L & 30 & $8292,55 * *$ & $8293,29 * *$ & $8293,32 * *$ & $8293,29 * *$ \\
Erro 2 & 140 & 3734,00 & 3734,11 & 3734,06 & 3734,07 \\
\hline Média (m ${ }^{3}$ sólido/ha) & & 500,59 & 628,55 & 628,55 & 628,55 \\
CV 1 (\%) & & 19,81 & 15,78 & 15,78 & 15,78 \\
CV 2 (\%) & 12,21 & 9,72 & 9,72 & 9,72 \\
\hline
\end{tabular}

${ }^{*} \mathrm{e}^{* *}$ significativos pelo teste $\mathrm{F}$ a 5 e $1 \%$ de probabilidade, respectivamente; e n.s.: não-significativo.

Pelos resultados obtidos, fica bem evidente que, em plantas perenes, é difícil utilizar uma expressão única para correção de estandes como a proposta para a cultura do milho por Zuber (1942). Isso porque, como se verificou, os clones diferem na capacidade de compensação. Esta também difere de acordo com as condições edafoclimáticas e com a idade de avaliação. Tal fato, inclusive, tem sido observado em plantas anuais. 
Considera-se que neste trabalho a amplitude de variação nas falhas foi perda de até $50 \%$ das plantas na parcela, o que, acredita-se, representa a maioria das situações. E, dada a diferença entre os clones avaliados e as condições ambientais, a inferência de que a análise de regressão deve ser utilizada para tornar comparável à dos clones em qualquer situação e facilitar a análise conjunta provavelmente possa ser extrapolada para outras condições, no Brasil.

\section{CONCLUSÕES}

- A maioria dos clones avaliados não compensa, em parte, a ausência das plantas vizinhas.

- A capacidade de compensação difere entre os clones e condições edafoclimáticas.

- Independentemente do clone e do local, a correção do estande por regressão foi eficiente para efetuar a seleção dos melhores clones, sendo mais expressiva com os dados de total de parcela.

\section{REFERÊNCIAS BIBLIOGRÁFICAS}

ANDRADE, H.B. et al. Avaliação de espécies e procedências de Eucalyptus L' Héritier (Myrtaceae) nas regiões norte e noroeste do Estado de Minas Gerais. Revista Árvore, v.18, n.3, p.215-229, 1994.

CRUZ, V.F. Estudo sobre a correção de produção de parcelas em ensaios com milho. 1971. 143f. Tese (Doutorado em Genética e Melhoramento) - Escola Superior de Agricultura Luiz de Queiroz, Piracicaba, 1971.

FERNANDES, M.I.P.S.; RAMALHO, M.A.P.; LIMA, P.C. Comparação de métodos de correção em estandes de feijão. Pesquisa Agropecuária Brasileira, v.24, n.8, p.997-1002, 1989.
GOLFARI, L. Zoneamento ecológico do Estado de Minas Gerais para reflorestamento. Rio de Janeiro: PRODEPEF, 1975. 65p. (Série Técnica, 3).

GOMES, F.P. Curso de estatística experimental. 12. ed. Piracicaba: Nobel. 1987. 467p.

MARQUES JÚNIOR, O.G. Eficiência de experimentos com a cultura do feijão. 1997. 80p. Tese (Doutorado em Genética e Melhoramento de Plantas) - Universidade Federal de Lavras, Lavras, 1997.

RAMALHO, M.A.P; FERREIRA, D.F.; OLIVEIRA, A.C. de. Experimentação em genética e melhoramento de plantas. Lavras: Universidade Federal de Lavras, 2000. 303p.

SCHMILDT, E.R. Correção de rendimento de parcelas, estratificação ambiental e adaptabilidade e estabilidade de cultivares de milho. 2000. 110f. Tese (Doutorado em Genética e Melhoramento de Plantas) -

Universidade Federal de Viçosa, Viçosa, 2000.

STEEL, R.G.D.; TORRIE, J.H.; DICKEY, D.A. Principles and procedures of statistics: a Biometrical Approach. 3. ed. New York: Mc Graw Hill, 1997. 666p.

VENCOVSKY, R.; BARRIGA, P. Genética biométrica no fitomelhoramento. Ribeirão Preto: Sociedade Brasileira de Genética, 1992. 486p.

VERONESI, J.A. et al. Comparação de métodos de ajuste do rendimento de parcelas com estandes variados. Pesquisa Agropecuária Brasileira, v.30, n.2, p.169-174, 1995.

ZUBER, M.S. Relative efficiency of incomplete block designs using corn uniform trial data. Journal of the American Society of Agronomy, v.34, n.1, p.34-47, 1942. 